\title{
Research Article \\ Optimal Iterative Learning Fault-Tolerant Guaranteed Cost Control for Batch Processes in the 2D-FM Model
}

\author{
Limin Wang ${ }^{1,2,3}$ and Weiwei Dong ${ }^{2}$ \\ ${ }^{1}$ College of Sciences, Liaoning Shihua University, Fushun 113001, China \\ ${ }^{2}$ Fok Ying Tung Graduate School, Hong Kong University of Science and Technology, Hong Kong \\ ${ }^{3}$ Department of Control Science and Engineering, Zhejiang University, Zhejiang 310027, China \\ Correspondence should be addressed to Limin Wang, wanglimin0817@163.com
}

Received 2 January 2012; Accepted 22 February 2012

Academic Editor: Pavel Drábek

Copyright (C) 2012 L. Wang and W. Dong. This is an open access article distributed under the Creative Commons Attribution License, which permits unrestricted use, distribution, and reproduction in any medium, provided the original work is properly cited.

\begin{abstract}
This paper develops the optimal fault-tolerant guaranteed cost control scheme for a batch process with actuator failures. Based on an equivalent two-dimensional Fornasini-Marchsini (2D-FM) model description of a batch process, the relevant concepts of the fault-tolerant guaranteed cost control are introduced. The robust iterative learning reliable guaranteed cost controller (ILRGCC), which includes a robust extended feedback control for ensuring the performances over time and an iterative learning control (ILC) for improving the tracking performance from cycle to cycle, is formulated such that it cannot only guarantee the closed-loop convergency along both the time and the cycle directions but also satisfy both the $H_{\infty}$ performance level and a cost function having upper bounds for all admissible uncertainties and any actuator failures. Conditions for the existence of the controller are derived in terms of linear matrix inequalities (LMIs), and a design procedure of the controller is presented. Furthermore, a convex optimization problem with LMI constraints is formulated to design the optimal guaranteed cost controller which minimizes the upper bound of the closed-loop system cost. Finally, an illustrative example of injection molding is given to demonstrate the effectiveness and advantages of the proposed $2 \mathrm{D}$ design approach.
\end{abstract}

\section{Introduction}

Though studies on batch process control can be dated back to 1930s [1], the last 10 years have witnessed a very widespread concern to batch processing technologies that is driven by the business of manufacturing [2]. The process optimization and control lag far behind the development of a continuous production process. Advanced control has critical importance to the quality and quality consistency of batch processes because batch processes are 
the preferred manufacturing choice for low-volume and high-value products. It is necessary to study the advanced control problem for the batch process.

Meanwhile, the demand for productivity leads increasingly for the chemical plant to operate under challenging conditions, which consequently exposes the possibility of system failures. A chemical process typically has a large number of measurements and actuators. If a failure is not controlled promptly with a proper corrective action, it will degrade the process performance and even result in safety problems for the plant and personnel. Therefore, from safety as well as performance point of view, it is interesting in studying the problem of fault-tolerant control (FTC) for the system with actuator failures. Reliable control is a popular FTC method [3-5]. Recently, the study of reliable control has received considerable attention because of the growing demands on reliability. However, most of them are designed for continuous processes. Batch processes are also suffering from this problem. Due to the complexity of the batch process, the harm that it suffers is more serious than that a continuous process does. As early as 1982, Wensley pointed out the importance and urgency of faulttolerant control for batch processes. But, as supporting technology is not yet mature, only limited results on FTC have been available up to now [6-8].

To exploit the repetitive nature of batch processes, iterative learning control (ILC) has been used widely. In [9], a robust ILC was proposed for an injection mold process with uncertain initial resetting and disturbances, and the output can track an arbitrary bounded reference. A systematic method for the analysis and design of ILC systems was presented in [10], in regard to the frequency domain. A model-based ILC strategy for the tracking control of product quality in batch processes was proposed in [11]. Presently, Shi et al. proposed a more general design framework for ILC of batch process: they first proposed the feedback integrated with ILC scheme for the batch process; then, the batch process under ILC is modeled as a two-dimensional (2D) system and the design of a robust ILC for a batch process is transformed to a robust stabilization problem of the 2D system [1217]. The aforementioned results are obtained in the normal case. According to faulty cases, for example, actuator failures, Wang et al. [6] developed a 2D iterative learning reliable control (ILRC) for batch processes in a 2D Fornasini-Marchsini (2D-FM) model, and the ILRC controller is designed to guarantee the closed-loop fault-tolerant system's stability in terms of linear matrix inequality (LMI); in view of sensor failures, the main results in robust fault-tolerant control of batch processes are introduced in two classes: passive and active $[7,8]$.

In fact, there are two major issues in the robust controller design. The first is concerned with the robust stability of the uncertain closed-loop system, and the other is the robust performance. Note that the latter is more important since when controlling a system dependent on uncertain parameters, it is always desirable to design a control system which is not only stable but also guarantees an adequate level of performance. Since the so-called guaranteed cost control approach first introduced by Chang and Peng [18], this issue on one-dimensional (1D) systems has been addressed extensively. In recent years, 2D discrete systems have found various applications in many areas. The guaranteed cost control problem for 2D discrete uncertain systems has received considerable attention and a robust controller design method has been established. Furthermore, an optimization problem is introduced to select the suboptimal guaranteed cost controller which minimizes the upper bound of the cost function [19-21]. Although the results can be extended to robust iterative learning guaranteed cost controller design, the resulted ILC law will be dependent on a state feedback. On the other hand, no results on robust iterative learning fault-tolerant guaranteed cost control have been available up to now. 
In this paper, according to actuator failures, the authors use the 2D theory to model, analyze, and design robust iterative learning fault-tolerant control (ILFTC) system. We first propose a $2 \mathrm{D}$ controller, which essentially consists of two types of controls: one is robust feedback control with extended information, using the real-time information to ensure the robust control performances along time, and the other is ILC, improving the tracking performance from cycle to cycle. Then we establish a 2D-FM model serving as a process model for the proposed design. Thus, sufficient conditions for the existence of the robust optimal ILRGCC are constructed. Further, a convex optimization problem is introduced to find the controller which minimizes the upper bound on the cost function. In addition, to solve the nonrepeatable perturbation, the control law will satisfy the $H_{\infty}$ performance. The main merits of this work can be summarized on two perspectives. (i) An ILFTC system is represented equivalently as a $2 \mathrm{D}$ model. Based on this, the robust optimal guaranteed cost control that are related to the ILFTC system can be translated to optimal guaranteed cost control that are related to a 2D system. This provides a foundation for the design and analysis of an ILFTC system based on a 2D system. (ii) Robust optimal fault-tolerant guaranteed cost control along different axes introduced for the 2D system provides a powerful tool for analyzing robust optimal guaranteed cost control of the ILC system over time and cycles. Simulation with injection pressure control shows that the proposed 2D-ILRGCC design method achieves the design objectives well.

Throughout this paper, the following notations are used: $R^{n}$ represents Euclidean $n$ space, with the norm denoted by $\|\cdot\|$. For any matrix $M, M>0(M<0)$ means $M$ is a positive (negative) definite matrix. $M^{T}$ represents the transpose of matrix $M . I$ and 0 , respectively, denote the identity matrix and the zero matrix with appropriate dimensions. The asterisk notation $(*)$ represents the symmetric element of a matrix. $|\cdot|$ denotes absolute value of ".".

For a 2D signal $\omega(t, k)$, if $\|\omega(\cdot, \cdot)\|_{2 \mathrm{D}-2 e}=\sqrt{\sum_{t=0}^{N_{1}} \sum_{k=0}^{N_{2}}\|\omega(\cdot, \cdot)\|^{2}}<\infty$ for any integers $N_{1}$, $N_{2}>0$, then $\omega(t, k)$ is said to be in $\ell_{2 \mathrm{D}-2 e}$ space, as denoted by $\omega(\cdot, \cdot) \in \ell_{2 \mathrm{D}-2 e}$.

\section{Problem Description}

Consider process $\sum_{P}$, repetitively performing a task over a certain period of time (called a cycle), described by the following discrete-time model with uncertain parameter perturbations:

$$
\begin{aligned}
& \sum_{P}:\left\{\begin{array}{l}
x(t+1, k)=\left(A+\Delta_{a}(t, k)\right) x(t, k)+B u(t, k) \\
y(t, k)=C x(t, k)
\end{array}\right. \\
& \text { for } x(0, k)=x_{0, k} ; t=0,1,2, \ldots, T ; k=1,2, \ldots,
\end{aligned}
$$

where $k$ indicates the cycle; $t$ is the time index; $x(t, k) \in R^{n}, y(t, k) \in R^{l}$, and $u(t, k) \in R^{m}$ are, respectively, the state, output, and input of the process at time $t$ in the $k$ th cycle; $x_{0, k}$ is the initial condition of the $k$ th cycle. $\{A, B, C\}$ are constant matrices of appropriate dimensions, and $\Delta_{a}(t, k)$ denotes some perturbations at time $t$ in the $k$ th cycle and may be specified as $\Delta_{a}(t, k)=E \Delta(t, k) F$ with $\Delta^{T}(t, k) \Delta(t, k) \leq I, 0 \leq t \leq T, k=1,2, \ldots$, where $\{E, F\}$ are known constant matrices. $\Delta(t, k)$ is generally represented as the functions of both time $t$ and cycle $k$. If $\Delta(t, k)$ depends on time $t$ only, then the uncertain parameter perturbations are called repeatable, otherwise nonrepeatable. For any two sequential cycles, $\delta\left(\Delta_{a}(t, k)\right)=\Delta_{a}(t, k)-$ $\Delta_{a}(t, k-1)$ denotes the cycle-to-cycle parameter perturbation. 
For control input $u_{i}(t, k)$ (where $i=1,2, \ldots, m$ ), let $u_{i}^{F}(t, k)$ denote the signal from the failed actuator. The following failure model is expressed as

$$
u_{i}^{F}(t, k)=\alpha_{i} u_{i}(t, k) \quad(\text { for } i=1,2, \ldots, m),
$$

where

$$
0 \leq \underline{\alpha}_{i} \leq \alpha_{i} \leq \bar{\alpha}_{i} \quad(\text { for } i=1,2, \ldots, m)
$$

The terms $\underline{\alpha}_{i}\left(\underline{\alpha}_{i} \leq 1\right)$ and $\bar{\alpha}_{i}\left(\bar{\alpha}_{i} \leq 1\right)$ are known scalars.

Denote

$$
\begin{aligned}
u^{F} & =\left[u_{1}^{F}, u_{2}^{F}, \ldots, u_{m}^{F}\right]^{T}, \\
\bar{\alpha} & =\operatorname{diag}\left[\bar{\alpha}_{1}, \bar{\alpha}_{2}, \ldots, \bar{\alpha}_{m}\right], \\
\underline{\alpha} & =\operatorname{diag}\left[\underline{\alpha}_{1}, \underline{\alpha}_{2}, \ldots, \underline{\alpha}_{m}\right], \\
\alpha & =\operatorname{diag}\left[\alpha_{1}, \alpha_{2}, \ldots, \alpha_{m}\right] .
\end{aligned}
$$

Hence, a batch process with state delay and actuator failures can be described by

$$
\begin{aligned}
\sum_{P-F}: & x(t+1, k)=\left(A+\Delta_{a}(t, k)\right) x(t, k)+B \alpha u(t, k), \\
y(t, k) & =C x(t, k), \\
x(t, k) & =x_{0, k} ; \quad t=0,1,2, \ldots, T ; k=1,2, \ldots
\end{aligned}
$$

The control objective is to design a fault-tolerant guaranteed cost control law such that the output of the process tracks a given trajectory, $y_{d}(t)$, as closely as possible, even with actuator failures. Introduce the following notations:

$$
\begin{gathered}
\beta=\operatorname{diag}\left[\beta_{1}, \beta_{2}, \ldots, \beta_{m}\right], \\
\beta_{0}=\operatorname{diag}\left[\beta_{10}, \beta_{20}, \ldots, \beta_{m 0}\right],
\end{gathered}
$$

with

$$
\beta_{i}=\frac{\bar{\alpha}_{i}+\underline{\alpha}_{i}}{2}, \quad \beta_{i 0}=\frac{\bar{\alpha}_{i}-\underline{\alpha}_{i}}{\bar{\alpha}_{i}+\underline{\alpha}_{i}} \quad(\text { for } i=1,2, \ldots, m)
$$

From (2.4) and (2.6a)-(2.6c), we know that there exists an unknown matrix $\alpha_{0}$ such that

$$
\alpha=\left(I+\alpha_{0}\right) \beta
$$


with

$$
\left|\alpha_{0}\right| \leq \beta_{0} \leq I
$$

where

$$
\alpha_{0} \triangleq \operatorname{diag}\left[\alpha_{01}, \alpha_{02}, \ldots, \alpha_{0 m}\right], \quad\left|\alpha_{0}\right| \triangleq \operatorname{diag}\left[\left|\alpha_{01}\right|,\left|\alpha_{02}\right|, \ldots,\left|\alpha_{0 m}\right|\right]
$$

\section{Iterative Learning Reliable Guaranteed Cost Control}

\subsection{Equivalent 2D Model Representation}

For process $\sum_{P}$, use an ILC law with the following form:

$$
\sum_{\text {ilc }}: u(t, k)=u(t, k-1)+r(t, k) \quad(\text { for } u(t, 0)=0, t=0,1,2, \ldots, T),
$$

where $u(t, 0)$ is the initial value of iteration and $r(t, k) \in R^{m}$ is called the updating law of the ILC to be determined. The objective for ILC design is to establish a procedure for the design of optimal guaranteed cost controller (3.1) (or equivalent updating law $r(t, k)$ ) such that $y(t, k)$ tracks $y_{d}(t)$ as well as guarantees the closed-loop system preserving an adequate control performance.

Design

$$
\begin{aligned}
\delta_{k}(f(t, k)) & =f(t, k)-f(t, k-1), \\
e(t, k) & =y(t, k)-y_{d}(t) .
\end{aligned}
$$

It follows from (2.1) along with the definition of (3.2) that

$$
\begin{gathered}
\delta_{k}(x(t+1, k))=\left(A+\Delta_{a}(t, k)\right) \delta_{k}(x(t, k))+B \alpha r(t, k)+\omega(t, k), \\
e(t+1, k)=y(t+1, k)-y_{d}(t)=e(t+1, k-1)+C \delta_{k}(x(t+1, k)) \\
=e(t+1, k-1)+C\left(A+\Delta_{a}(t, k)\right) \delta_{k}(x(t, k))+C B \alpha r(t, k)+C \omega(t, k),
\end{gathered}
$$

where

$$
\omega(t, k)=\delta_{k}\left(\Delta_{a}(t, k)\right) x(t, k-1)
$$

Obviously, for repeatable parameter perturbations, $\omega(t, k)=0$; for a nonrepeatable disturbance, $\omega(t, k) \neq 0$.

On the other hand, for the ILC scheme, the use of more learning information may lead to a better control performance. An advantage of using the design framework proposed in 
this paper is that the learning information used by the ILC law can be flexibly extended, along the time and/or the cycle. In order to achieve steady-state tracking error along the time direction to be of fast convergence, here assume that the general model of the feedback/feedforward control to be extended is described by the following linear dynamical model:

$$
\sum_{e}: x_{e}(t+1, k)=A_{e} x_{e}(t, k)+B_{e} e(t, k)
$$

where $x_{e}(t, k)$ is an extended state dynamically determined by $e(t, k)$ and $\left\{A_{e}, B_{e}\right\}$ are parameters that are specified based on the structure of the feedback and/or feed-forward controls to be extended. In this paper, an integral feedback control can be extended by simply specifying $A_{e}=B_{e}=I$.

Thereby, an equivalent $2 \mathrm{D}$ system description of the previous batch process, combination of the extended model $\sum_{e}$ with (3.3a)-(3.3c), can be expressed as

$$
\sum_{2 \mathrm{D}-e p-F}:\left\{\begin{aligned}
& X(t+1, k)=\left(A_{1}+\Delta A_{1}(t, k)\right) X(t, k)+A_{2} X(t+1, k-1)+\bar{B} \alpha r(t, k)+G \omega(t, k) \\
&=A_{1}(t, k) X(t, k)+A_{2} X(t+1, k-1)+\bar{B} \alpha r(t, k)+G \omega(t, k), \\
& Z(t, k) \triangleq e(t, k)=H X(t, k),
\end{aligned}\right.
$$

where

$$
\begin{gathered}
X(t, k)=\left[\begin{array}{c}
\delta_{k}(x(t, k)) \\
x_{e}(t, k) \\
e(t, k)
\end{array}\right], \quad A_{1}=\left[\begin{array}{ccc}
A & 0 & 0 \\
0 & I & I \\
C A & 0 & 0
\end{array}\right], \quad A_{2}=\left[\begin{array}{lll}
0 & 0 & 0 \\
0 & 0 & 0 \\
0 & 0 & I
\end{array}\right], \quad \bar{B}=\left[\begin{array}{c}
B \\
0 \\
C B
\end{array}\right], \\
\Delta A_{1}(t, k)=\left[\begin{array}{ccc}
\Delta_{a}(t, k) & 0 & 0 \\
0 & 0 & 0 \\
C \Delta_{a}(t, k) & 0 & 0
\end{array}\right], \quad \Delta A_{1}(t, k)=\bar{E} \Delta(t, k) \bar{F}, \quad \bar{E}=\left[\begin{array}{c}
E \\
0 \\
C E
\end{array}\right], \\
\bar{F}=\left[\begin{array}{lll}
F & 0 & 0
\end{array}\right], \quad G=\left[\begin{array}{l}
I \\
0 \\
C
\end{array}\right], \quad H=\left[\begin{array}{lll}
0 & 0 & I
\end{array}\right] .
\end{gathered}
$$

Model $\sum_{2 \mathrm{D}-e p-F}$ is a typical 2D-FM model with uncertain perturbations; because this model equivalently represents the dynamical behavior of the tracking error of the system (2.1), it is called the equivalent 2D tracking error model of system (2.1). Therefore, it is clear that the design of the updating law $r(t, k)$ for system (2.1) is equivalent to the design of a fault-tolerant guaranteed cost control law for the equivalent 2D tracking error model $\sum_{2 \mathrm{D}-e p-F}$. Design a controller as follows:

$$
r(t, k)=K\left[\begin{array}{c}
X(t, k) \\
X(t+1, k-1)
\end{array}\right]=\left[\begin{array}{ll}
K_{1} & K_{2}
\end{array}\right]\left[\begin{array}{c}
X(t, k) \\
X(t+1, k-1)
\end{array}\right] .
$$


The closed-loop 2D-FM system then is given by

$$
\sum_{2 \mathrm{D}-e p-F-C}:\left\{\begin{array}{l}
X(t+1, k)=A_{1 k} X(t, k)+A_{2 k} X(t+1, k-1)+G \omega(t, k) \\
Z(t, k) \triangleq e(t, k)=H X(t, k)
\end{array}\right.
$$

where $A_{1 k}=A_{1}(t, k)+\bar{B} \alpha K_{1}$ and $A_{2 k}=A_{2}+\bar{B} \alpha K_{2}$.

For 2D system $\sum_{2 \mathrm{D}-e p-F}$, assume that it has a finite set of initial conditions that is, there exists two positive integers $t, k$ such that

$$
X(t, 0)=0, \quad t \geq r_{1} ; \quad X(0, k)=0, \quad k \geq r_{2},
$$

where $r_{1}<\infty$ and $r_{2}<\infty$ are positive integers. Here $X(t, 0)$ and $X(0, k)$ are called $K$-boundary and $T$-boundary, respectively, and the initial boundary conditions are arbitrary but belong to the set

$$
S=\left\{X(t, 0), X(0, k) \in R^{n}: X(t, 0)=\Theta v_{1}, X(0, k)=\Theta v_{2}, v_{t}{ }^{T} v_{t} \leq I,(t=1,2)\right\},
$$

where $\Theta$ is a given matrix.

Associated with 2D system (3.5) is the following cost function:

$$
\begin{aligned}
J & =\sum_{t=0}^{N_{1}} \sum_{k=1}^{N_{2}}\left[X^{T}(t, k) U_{1} X(t, k)+X^{T}(t+1, k-1) U_{2} X(t+1, k-1)+r^{T}(t, k) U_{3} r(t, k)\right] \\
& =\sum_{t=0}^{N_{1}} \sum_{k=1}^{N_{2}} \varphi^{T}(t, k)\left[\begin{array}{cc}
U_{1}+K_{1}^{T} U_{3} K_{1} & K_{1}^{T} U_{3} K_{2} \\
K_{2}^{T} U_{3} K_{1} & U_{2}+K_{2}^{T} U_{3} K_{2}
\end{array}\right] \varphi(t, k),
\end{aligned}
$$

where $U_{1}>0, U_{2}>0, U_{3}>0$, and $\varphi^{T}(t, k)=\left[X(t, k) X^{T}(t+1, k-1)\right]$.

Introduce the following definitions to establish a procedure for the design of updating law $r(t, k)$ which guarantees the closed-loop system both robust stable and preserving a adequate control performance.

Definition 3.1. Denote $X_{r}=\sup \{\|X(t, k)\|: t+k=r, t, k \geq 1\}$; consider the uncertain 2D system (3.5) and the cost function (3.10); for any bounded boundary conditions satisfying (3.9b), all admissible uncertainties, and any admissible actuator faults, if there exists a controller $r^{*}(t, k)$ and some specified constant $J^{*}$ such that the state of the resulting closed-loop system (3.8) satisfies $\lim _{r \rightarrow \infty} X_{r}=0$ and its cost function (3.10) satisfies $J \leq J^{*}$, then $J^{*}$ is said to be a faulttolerant guaranteed cost and $r^{*}(t, k)$ is said to be a fault-tolerant guaranteed cost control law for the uncertain 2D system (3.5).

Definition 3.2. Assume that the $K$-boundary $X(t, 0)=0$; for any $T$-boundary $X(0, k) \in S$, any integer $N>0$, all admissible uncertainties, and any admissible actuator faults, if there exists a controller $r^{*}(t, k)$ and some specified constant $\bar{J}_{1}^{*}$ such that the resulting closed-loop system (3.8) satisfies $\lim _{t \rightarrow \infty} \sum_{k=1}^{N}\left\|X_{r}\right\|=0$ and its cost function (3.10) satisfies $J \leq \bar{J}_{1}^{*}$, then $\bar{J}_{1}^{*}$ is said 
to be a $T$-fault-tolerant guaranteed cost and $r^{*}(t, k)$ is said to be a $T$-fault-tolerant guaranteed cost controller for the 2D system (3.5).

Definition 3.3. Assume that the $T$-boundary $X(0, k)=0$. For any $K$-boundary $X(t, 0) \in S$, any integer $N>0$, all admissible uncertainties, and any admissible actuator faults, if there exists a controller $r^{*}(t, k)$ and some specified constant $\bar{J}_{2}^{*}$ such that the resulting closed-loop system (3.8) satisfies $\lim _{k \rightarrow \infty} \sum_{t=1}^{N}\left\|X_{r}\right\|=0$ and its cost function (3.10) satisfies $J \leq \bar{J}_{2}^{*}$, then $\bar{J}_{2}^{*}$ is said to be a $K$-fault-tolerant guaranteed cost and $r^{*}(t, k)$ is said to be a $K$-fault-tolerant guaranteed cost controller for the 2D system (3.5).

Definition 3.4. For a given scalar $\gamma>0$, control law $r^{*}(t, k)$ is a robust $H_{\infty}$ fault-tolerant guaranteed cost control law for the uncertain 2D system (3.5); if the following conditions hold for all admissible parameter uncertainties:

(1) the resulting closed-loop system (3.8) with $\omega(t, k)=0$ is asymptotically stable;

(2) with the zero initial condition, the controlled output $z(t, k)$ satisfies

$$
\|z\|_{2 \mathrm{D}-2 e} \leq r\|\omega\|_{2 \mathrm{D}-2 e}
$$

(3) in the case when $\omega(t, k)=0$, the cost function for the resulting closed-loop system (3.8) satisfies $J \leq J^{*}$.

Lemma 3.5. The $2 D$ closed-loop system $\sum_{2 D-e p-F-C}$ is $2 D$-fault-tolerant guaranteed cost control if there is a function $V(\cdot)$ that satisfies the following conditions:

(a) $V(x) \geq 0$ for $x \in R^{n}$, and $V(x)=0 \leftrightarrow x=0$;

(b) $V(x) \rightarrow \infty$ as $\|x\| \rightarrow \infty$;

(c) for any boundary conditions, any admissible actuator faults satisfy (2.3) and for all $T_{0}>$ $0, K_{0}>0, i>0$,

$$
\sum_{\substack{t+k=T_{0}+K_{0}+i+1 \\ T_{0} \leq \leq \leq T_{0}+i \\ K_{0} \leq k \leq K_{0}+i}} V(x(t, k))<\sum_{\substack{t+k=T_{0}+K_{0}+i \\ T_{0} \leq \leq \leq T_{0}+i \\ K_{0} \leq k \leq K_{0}+i}} V(x(t, k)) .
$$

Similar results can be obtained for $T$-fault-tolerant guaranteed cost control and $K$ fault-tolerant guaranteed cost control.

Lemma 3.6. The $2 D$ closed-loop system $\sum_{2 D-e p-F-C}$ is T-fault-tolerant guaranteed cost control if there is a function $V(\cdot)$ that satisfies the following conditions:

(a) $V(x) \geq 0$ for $x \in R^{n}$, and $V(x)=0 \leftrightarrow x=0$;

(b) $V(x) \rightarrow \infty$ as $\|x\| \rightarrow \infty$; 
(c) for any $T$-boundary $X(0, k)$, any integer $N>0$, a zero K-boundary $X(t, 0)$, any admissible actuator faults satisfy (2.3) and for all $t>0$

$$
\sum_{k=1}^{N} V(x(t+1, k))<\sum_{k=1}^{N} V(x(t+1, k)) .
$$

Lemma 3.7. The $2 D$ closed-loop system $\sum_{2 D-e p-F-C}$ is $K$-fault-tolerant guaranteed cost control if there is a function $V(\cdot)$ that satisfies the following conditions:

(a) $V(x) \geq 0$ for $x \in R^{n}$, and $V(x)=0 \leftrightarrow x=0$;

(b) $V(x) \rightarrow \infty$ as $\|x\| \rightarrow \infty$;

(c) for any $K$-boundary $X(t, 0)$, any integer $N>0$, a zero $T$-boundary $X(0, k)$, any admissible actuator faults satisfy (2.3) and for all $k>0$

$$
\sum_{t=1}^{N} V(x(t, k+1))<\sum_{t=1}^{N} V(x(t, k))
$$

\subsection{Reliable Guaranteed Cost Controller Design and System Structure}

In this section, we will design a reliable updating law $r(t, k)$ such that the resulting closedloop system (3.8) is 2D-fault-tolerant guaranteed cost control and the cost function of closedloop system is lower than a specified upper bound.

Lemma 3.8 (see [22]). For any matrices $D \in R^{n \times n_{f}}, E \in R^{n_{f} \times n}$, and $F \in R^{n_{f} \times n_{f}}$ with $\|F\| \leq 1$, and scalar $\varepsilon>0$, the following inequality holds:

$$
D F E+E^{T} F^{T} D^{T} \leq \varepsilon^{-1} D D^{T}+\varepsilon E^{T} E .
$$

Lemma 3.9 ((Schur Complement) [23]). Assume that $W, L$, and $V$ are given matrices with appropriate dimensions, where $W$ and $V$ are positive definite matrices; then

$$
L^{T} V L-W<0
$$

if and only if

$$
\left[\begin{array}{cc}
-W & L^{T} \\
L & -V^{-1}
\end{array}\right]<0 \quad \text { or } \quad\left[\begin{array}{cc}
-V^{-1} & L \\
L^{T} & -W
\end{array}\right]<0
$$

Theorem 3.10. Consider the 2D system (3.5) with the initial condition (3.9a)-(3.9b) and cost function (3.10); assume $\omega(t, k)=0$; if there exist positive definite symmetric (PDS) matrices $S_{1}, S_{2}, \Omega \in R^{(n+l) \times(n+l)}$, matrices $Y_{1}, Y_{2} \in R^{m \times(n+l)}$, and positive scalars $\varepsilon_{i}(i=1,2)$ such that the 
following LMI (3.18) holds, then there exists a $2 D$ static-state feedback controller $r(t, k)=K_{1} X$ $(t, k)+K_{2} X(t+1, k-1)$ that solves the addressed robust guaranteed cost control problem:

$$
\left[\begin{array}{cccccccc}
-S_{1} & 0 & \Omega A_{1}^{T}+Y_{1}^{T} \beta \bar{B}^{T} & Y_{1}^{T} & Y_{1}^{T} \beta & \Omega \bar{F}^{T} & \Omega & 0 \\
* & -S_{2} & \Omega A_{2}^{T}+Y_{2}^{T} \beta \bar{B}^{T} & Y_{2}^{T} & Y_{2}^{T} \beta & 0 & 0 & \Omega \\
* & * & -\Omega+\varepsilon_{1} \bar{B} \beta_{0}^{2} \bar{B}^{T}+\varepsilon_{2} \overline{E E}^{T} & 0 & 0 & 0 & 0 & 0 \\
* & * & * & -U_{3}^{-1} & 0 & 0 & 0 & 0 \\
* & * & * & * & -\varepsilon_{1} I & 0 & 0 & 0 \\
* & * & * & * & * & -\varepsilon_{2} I & 0 & 0 \\
* & * & * & * & * & * & -U_{1}^{-1} & 0 \\
* & * & * & * & * & * & * & -U_{2}^{-1}
\end{array}\right]<0 .
$$

In this situation, a suitable control law is given by

$$
K=\left[K_{1} K_{2}\right]=\left[\Upsilon_{1} \Omega^{-1} Y_{2} \Omega^{-1}\right]
$$

Moreover, the corresponding cost function of the resulting closed-loop $2 D$ system $\sum_{2 D-e p-F-C}$ satisfies

$$
J \leq \sum_{k=1}^{N_{2}} X^{T}(0, k) P S_{1} P x X(0, k)+\sum_{t=0}^{N_{1}} X^{T}(t+1,0) P S_{2} P X(t+1,0)=J^{*} .
$$

Proof. Design the following quadratic Lyapunov function:

$$
V_{[\cdot]}(X(t, k))=\|X(t, k)\|_{[\cdot]} \triangleq X^{T}(t, k)[\cdot] X(t, k) \text {, }
$$

where [.] is any PDS matrix with appropriate dimensions. For PDS matrices $P, Q_{1}$, and $Q_{2}$, all functions $V_{P}(\cdot), V_{Q_{1}}(\cdot)$, and $V_{Q_{2}}(\cdot)$ satisfy conditions (a) and (b) of Lemmas 3.5, 3.6, and 3.7. Because $\omega(t, k)=0$, we have

$$
\begin{aligned}
\Delta V(X(t, k)) & =V_{P}(X(t+1, k))-V_{Q_{1}}(X(t, k))-V_{Q_{2}}(X(t+1, k-1)) \\
& =\|X(t+1, k)\|_{P}-\|X(t, k)\|_{Q_{1}}-\|X(t+1, k-1)\|_{Q_{2}} .
\end{aligned}
$$

Along the trajectory of the closed-loop system (3.8), we obtain

$$
\begin{aligned}
& \Delta V(X(t, k))+\varphi^{T}(t, k)\left[\begin{array}{cc}
U_{1}+K_{1}^{T} U_{3} K_{1} & K_{1}^{T} U_{3} K_{2} \\
K_{2}^{T} U_{3} K_{1} & U_{2}+K_{2}^{T} U_{3} K_{2}
\end{array}\right] \varphi(t, k) \\
& \quad=\varphi^{T}(t, k)\left(\left[\begin{array}{cc}
-Q_{1} & 0 \\
0 & -Q_{2}
\end{array}\right]+\left[\begin{array}{c}
A_{1 k}^{T} \\
A_{2 k}^{T}
\end{array}\right] P\left[\begin{array}{ll}
A_{1 k} & A_{2 k}
\end{array}\right]+\left[\begin{array}{cc}
U_{1}+K_{1}^{T} U_{3} K_{1} & K_{1}^{T} U_{3} K_{2} \\
K_{2}^{T} U_{3} K_{1} & U_{2}+K_{2}^{T} U_{3} K_{2}
\end{array}\right]\right) \varphi(t, k) .
\end{aligned}
$$


Pre- and postmultiplying the left-hand side matrix in (3.18) by the matrix $\operatorname{diag}[P, P, I, I$, $I, I, I, I]$, respectively, it follows that the matrix inequality (3.18) is equivalent to

$$
\left[\begin{array}{cccccccc}
-P S_{1} P & 0 & P \Omega A_{1}^{T}+P Y_{1}^{T} \beta \bar{B}^{T} & P Y_{1}^{T} & P Y_{1}^{T} \beta & P \Omega \bar{F}^{T} & P \Omega & 0 \\
* & -P S_{2} P & P \Omega A_{2}^{T}+P Y_{2}^{T} \beta \bar{B}^{T} & P Y_{2}^{T} & P Y_{2}^{T} \beta & 0 & 0 & P \Omega \\
* & * & -\Omega+\varepsilon_{1} \bar{B} \beta_{0}^{2} \bar{B}^{T}+\varepsilon_{2} \overline{E E}^{T} & 0 & 0 & 0 & 0 & 0 \\
* & * & * & -U_{3}^{-1} & 0 & 0 & 0 & 0 \\
* & * & * & * & -\varepsilon_{1} I & 0 & 0 & 0 \\
* & * & * & * & * & -\varepsilon_{2} I & 0 & 0 \\
* & * & * & * & * & * & -U_{1}^{-1} & 0 \\
* & * & * & * & * & * & * & -U_{2}^{-1}
\end{array}\right]<0 .
$$

Using the definitions $\Omega=P^{-1}, S_{1}=P^{-1} Q_{1} P^{-1}, S_{2}=P^{-1} Q_{2} P^{-1}, Y_{1}=K_{1} P^{-1}$, and $Y_{2}=K_{2} P^{-1}$, and then using Lemmas 3.8 and 3.9, we conclude that (3.24) is equivalent to

$$
\left[\begin{array}{cc}
-Q_{1} & 0 \\
0 & -Q_{2}
\end{array}\right]+\left[\begin{array}{c}
A_{1 k}^{T} \\
A_{2 k}^{T}
\end{array}\right] P\left[\begin{array}{ll}
A_{1 k} & A_{2 k}
\end{array}\right]+\left[\begin{array}{cc}
U_{1}+K_{1}^{T} U_{3} K_{1} & K_{1}^{T} U_{3} K_{2} \\
K_{2}^{T} U_{3} K_{1} & U_{2}+K_{2}^{T} U_{3} K_{2}
\end{array}\right]<0
$$

So, we have

$$
\Delta V(X(t, k))+\varphi^{T}(t, k)\left[\begin{array}{cc}
U_{1}+K_{1}^{T} U_{3} K_{1} & K_{1}^{T} U_{3} K_{2} \\
K_{2}^{T} U_{3} K_{1} & U_{2}+K_{2}^{T} U_{3} K_{2}
\end{array}\right] \varphi(t, k)<0 .
$$

It follows that

$$
\Delta V(X(t, k))<-\varphi^{T}(t, k)\left[\begin{array}{cc}
U_{1}+K_{1}^{T} U_{3} K_{1} & K_{1}^{T} U_{3} K_{2} \\
K_{2}^{T} U_{3} K_{1} & U_{2}+K_{2}^{T} U_{3} K_{2}
\end{array}\right] \varphi(t, k)
$$

Since $\left[\begin{array}{cc}U_{1}+K_{1}^{T} U_{3} K_{1} & K_{1}^{T} U_{3} K_{2} \\ K_{2}^{T} U_{3} K_{1} & U_{2}+K_{2}^{T} U_{3} K_{2}\end{array}\right]>0$, it means that the following inequality is effective:

$$
V_{P}(X(t+1, k))<V_{Q_{1}}(X(t, k))+V_{Q_{2}}(X(t+1, k-1)) .
$$

Without loss of generality, suppose that $Q_{1}=\lambda P, Q_{2}=(1-\lambda) P$; it has that $Q_{1}=P-Q_{2}$, which leads to

$$
V_{p}(X(t+1, k)) \leq V_{P}(X(t, k))-V_{Q_{2}}(X(t, k))+V_{Q_{2}}(X(t+1, k-1)) .
$$


For any integers $T_{0}, K_{0}, i>0$, the following inequalities hold:

$$
\begin{aligned}
V_{p}\left(X\left(T_{0}+1, K_{0}+i\right)\right) \leq & V_{P}\left(X\left(T_{0}, K_{0}+i\right)\right)-V_{Q_{2}}\left(X\left(T_{0}, K_{0}+i\right)\right)+V_{Q_{2}}\left(X\left(T_{0}+1, K_{0}+i-1\right)\right), \\
V_{p}\left(X\left(T_{0}+2, K_{0}+i-1\right)\right) \leq & V_{P}\left(X\left(T_{0}+1, K_{0}+i-1\right)\right)-V_{Q_{2}}\left(X\left(T_{0}+1, K_{0}+i-1\right)\right) \\
& +V_{Q_{2}}\left(X\left(T_{0}+2, K_{0}+i-2\right)\right) \\
& \vdots \\
V_{p}\left(X\left(T_{0}+i, K_{0}+1\right)\right) \leq & V_{P}\left(X\left(T_{0}+i-1, K_{0}+1\right)\right)-V_{Q_{2}}\left(X\left(T_{0}+i-1, K_{0}+1\right)\right)+V_{Q_{2}}\left(X\left(T_{0}+i, K_{0}\right)\right) .
\end{aligned}
$$

The sum of these inequalities leads to

$$
\begin{aligned}
\sum_{\substack{t+k=T_{0}+K_{0}+i+1 \\
T_{0} \leq t=T_{0}+i \\
K_{0} \leq k=K_{0}+i}} V(t, k) & \leq \sum_{\substack{t+k=T_{0}+K_{0}+i \\
T_{0} \leq t=T_{0}+i \\
K_{0} \leq k=K_{0}+i}} V(t, k)-V_{Q_{2}}\left(X\left(T_{0}, K_{0}+i\right)\right)+V_{Q_{2}}\left(X\left(T_{0}+i, K_{0}\right)\right)-V_{P}\left(X\left(T_{0}+i, K_{0}\right)\right) \\
& \leq \sum_{\substack{t+k=T_{0}+K_{0}+i \\
T_{0} \leq t+T_{0}+i \\
K_{0} \leq k=K_{0}+i}} V(t, k)-V_{Q_{2}}\left(X\left(T_{0}, K_{0}+i\right)\right)-V_{Q_{1}}\left(X\left(T_{0}+i, K_{0}\right)\right) \\
& \leq \sum_{\substack{t+k=T_{+}+K_{0}+i \\
T_{0} \leq t+T_{0}+i \\
K_{0} \leq k=K_{0}+i}} V(t, k) .
\end{aligned}
$$

It is clear that the sum of the Lyapunov functional value decreases along the state trajectories. We conclude from Definition 3.1 that $\lim _{t+k \rightarrow \infty} X(t, k) \rightarrow 0$ holds. Consequently, the system (3.5) is asymptotically stable. Moreover, condition (c) of Lemma 3.5 is satisfied, which implies that the resulting closed-loop system (3.8) is guaranteed cost control.

Since the inequality (3.27) holds, there can be obtained

$$
\sum_{t=0}^{N_{1}} \sum_{k=1}^{N_{2}} \Delta V(X(t, k)) \leq \sum_{t=0}^{N_{1}} \sum_{k=1}^{N_{2}}-\varphi^{T}(t, k)\left[\begin{array}{cc}
U_{1}+K_{1}^{T} U_{3} K_{1} & K_{1}^{T} U_{3} K_{2} \\
K_{2}^{T} U_{3} K_{1} & U_{2}+K_{2}^{T} U_{3} K_{2}
\end{array}\right] \varphi(t, k) .
$$

It follows from (3.32) and the definitions of $Q_{1}$ and $Q_{2}$ that

$$
\begin{gathered}
\sum_{k=1}^{N_{2}}\left(V_{Q_{1}}\left(N_{1}, k\right)-V_{Q_{1}}(0, k)\right)+\sum_{t=0}^{N_{1}}\left(V_{Q_{2}}\left(t+1, N_{2}\right)-V_{Q_{2}}(t+1,0)\right) \\
\leq \sum_{t=0}^{N_{1}} \sum_{k=1}^{N_{2}}-\varphi^{T}(t, k)\left[\begin{array}{cc}
Q_{1}+K_{1}^{T} U K_{1} & K_{1}^{T} U K_{2} \\
K_{2}^{T} U K_{1} & Q_{2}+K_{2}^{T} U K_{2}
\end{array}\right] \varphi(t, k) .
\end{gathered}
$$

For $N_{1}, N_{2} \rightarrow \infty$, it follows from Definition 3.1 and (3.9a)-(3.9b) that

$$
\sum_{t=0}^{N_{1}} \sum_{k=1}^{N_{2}} \varphi^{T}(t, k)\left[\begin{array}{cc}
U_{1}+K_{1}^{T} U_{3} K_{1} & K_{1}^{T} U_{3} K_{2} \\
K_{2}^{T} U_{3} K_{1} & U_{2}+K_{2}^{T} U_{3} K_{2}
\end{array}\right] \varphi(t, k) \leq \sum_{k=1}^{N_{2}} V_{Q_{1}}(0, k)+\sum_{t=0}^{N_{1}} V_{Q_{2}}(t+1,0) .
$$


Therefore, it follows from Definition 3.1 that the result of Theorem 3.10 is true. This completes the proof.

In addition, according to the definitions of $Q_{1}$ and $Q_{2}$ and (3.28), the following inequalities hold:

$$
\begin{aligned}
V_{Q_{1}}(X(t+1, N))+V_{Q_{2}}(X(t+1, N)) & \leq V_{Q_{1}}(X(t, N))+V_{Q_{2}}(X(t+1, N-1)), \\
V_{Q_{1}}(X(t+1, N-1))+V_{Q_{2}}(X(t+1, N-1)) & \leq V_{Q_{1}}(X(t, N-1))+V_{Q_{2}}(X(t+1, N-2)) \\
& \vdots \\
V_{Q_{1}}(X(t+1,1))+V_{Q_{2}}(X(t+1,1)) & \leq V_{Q_{1}}(X(t, 1))+V_{Q_{2}}(X(t+1,0)) .
\end{aligned}
$$

From Definition 3.2 and summing the previous inequalities, we obtain

$$
\sum_{k=1}^{N} V_{Q_{1}}(X(t+1, k)) \leq \sum_{k=1}^{N} V_{Q_{1}}(X(t, k))
$$

Obviously, the system (3.5) is T-asymptotically stable; condition (c) of Lemma 3.6 is satisfied, which means that the resulting closed-loop system (3.8) is T-guaranteed cost control. Using (3.25), for any integers $N_{1}, N_{2}>0$, from Definition 3.3 and (3.9a)-(3.9b), it leads to

$$
\sum_{t=0}^{N_{1}} \sum_{k=1}^{N_{2}} \varphi^{T}(t, k)\left[\begin{array}{cc}
U_{1}+K_{1}^{T} U_{3} K_{1} & K_{1}^{T} U_{3} K_{2} \\
K_{2}^{T} U_{3} K_{1} & U_{2}+K_{2}^{T} U_{3} K_{2}
\end{array}\right] \varphi(t, k) \leq \sum_{k=1}^{N_{2}} V_{h}(0, k)
$$

This completes the proof of T-guaranteed cost control. Similarly, K-guaranteed cost control can be easily proved. As we all know, the results of the previous discussion are about repetitive disturbances. The following conclusion is given based on nonrepetitive perturbations.

Theorem 3.11. The robust $H_{\infty}$ guaranteed cost control problem of the $2 D$ system (3.5) is solvable via a $2 D$ static-state feedback controller $r(t, k)=K_{1} X(t, k)+K_{2} X(t+1, k-1)$ if there exist PDS matrices $S_{1}, S_{2}, \Omega \in R^{(n+l) \times(n+l)}$, matrices $Y_{1}, Y_{2} \in R^{m \times(n+l)}$, and positive scalars $\varepsilon_{i}(i=1,2), \gamma$ such that the following LMI holds:

$$
\left[\begin{array}{cccccccccc}
-S_{1} & 0 & \Omega A_{1}^{T}+Y_{1}^{T} \beta \bar{B}^{T} & Y_{1}^{T} & Y_{1}^{T} \beta & \Omega \bar{F}^{T} & \Omega & 0 & \Omega H^{T} & 0 \\
* & -S_{2} & \Omega A_{2}^{T}+Y_{2}^{T} \beta \bar{B}^{T} & Y_{2}^{T} & Y_{2}^{T} \beta & 0 & 0 & \Omega & 0 & 0 \\
* & * & -\Omega+\varepsilon_{1} \bar{B} \beta_{0}^{2} \bar{B}^{T}+\varepsilon_{2} \overline{E E}^{T} & 0 & 0 & 0 & 0 & 0 & 0 & G \\
* & * & * & -U_{3}^{-1} & 0 & 0 & 0 & 0 & 0 & 0 \\
* & * & * & * & -\varepsilon_{1} I & 0 & 0 & 0 & 0 & 0 \\
* & * & * & * & * & -\varepsilon_{2} I & 0 & 0 & 0 & 0 \\
* & * & * & * & * & * & -U_{1}^{-1} & 0 & 0 & 0 \\
* & * & * & * & * & * & * & -U_{2}^{-1} & 0 & 0 \\
* & * & * & * & * & * & * & * & -\gamma I & 0 \\
* & * & * & * & * & * & * & * & * & -\gamma I
\end{array}\right]<0 .
$$


In this case, the robust $H_{\infty}$ guaranteed cost control law can be still chosen as (3.19) and the corresponding cost function of the resulting closed-loop $2 D$ system $\sum_{2 D-e p-F-C}$ (3.8) still satisfies (3.20).

Proof. To solve the $H_{\infty}$ guaranteed cost control scheme of the 2D system (3.5) with zero boundary conditions for any nonzero $\omega(t, k) \in l_{2}\{[0, \infty],[0, \infty]\}$, we define

$$
J_{1}=\Delta V(X(t, k))+\gamma^{-1} z^{T}(t, k) z(t, k)-\gamma \omega^{T}(t, k) \omega(t, k),
$$

where

$$
\Delta V(X(t, k))=V_{p}(X(t+1, k))-V_{Q_{1}}(X(t, k))-V_{Q_{2}}(X(t+1, k-1)) .
$$

For any integers $N_{1}, N_{2}>0$, from the definitions of $Q_{1}$ and $Q_{2}$, and the assumption that all boundary conditions of system $\sum_{2 \mathrm{D}-e p-F-C}$ are zeros, it is obtained that

$$
\begin{aligned}
\sum_{t=0}^{M_{1}} \sum_{k=1}^{M_{2}} \Delta V(X(t, k)) & =\sum_{t=0}^{M_{1}} \sum_{k=1}^{M_{2}}\left[V_{p}(X(t+1, k))-V_{Q_{1}}(X(t, k))-V_{Q_{2}}(X(t+1, k-1))\right] \\
& =\sum_{t=1}^{M_{1}} \sum_{k=1}^{M_{2}} V_{p-Q_{1}-Q_{2}}(X(t, k))+\sum_{k=1}^{M_{2}-1} V_{p-Q_{2}}\left(X\left(M_{1}+1, k\right)\right)+V_{p}\left(X\left(M_{1}+1, M_{2}\right)\right) \geq 0 .
\end{aligned}
$$

Then, for any nonzero $\omega(t, k) \in l_{2}\{[0, \infty],[0, \infty]\}$, it has

$$
\begin{aligned}
& \sum_{t=0}^{M_{1}} \sum_{k=1}^{M_{2}}\left[\gamma^{-1} z^{T}(t, k) z(t, k)-\gamma \omega^{T}(t, k) \omega(t, k)\right] \\
& \quad \leq \sum_{t=0}^{M_{1}} \sum_{k=1}^{M_{2}} J_{1}=\sum_{t=0}^{M_{1}} \sum_{k=1}^{M_{2}}\left[\gamma^{-1} z^{T}(t, k) z(t, k)-\gamma \omega^{T}(t, k) \omega(t, k)+\Delta V(X(t, k))\right]
\end{aligned}
$$

because

$$
\begin{aligned}
& r^{-1} z^{T}(t, k) z(t, k)-\gamma \omega^{T}(t, k) \omega(t, k)+\Delta V(X(t, k)) \\
& \quad=\left[\begin{array}{c}
\varphi(t, k) \\
\omega(t, k)
\end{array}\right]^{T} \bar{\Gamma}\left[\begin{array}{c}
\varphi(t, k) \\
\omega(t, k)
\end{array}\right]-\varphi^{T}(t, k)\left[\begin{array}{cc}
U_{1}+K_{1}^{T} U_{3} K_{1} & K_{1}^{T} U_{3} K_{2} \\
K_{2}^{T} U_{3} K_{1} & U_{2}+K_{2}^{T} U_{3} K_{2}
\end{array}\right] \varphi(t, k),
\end{aligned}
$$


where

$$
\begin{aligned}
\bar{\Gamma}= & {\left[\begin{array}{ccc}
-Q_{1}+\gamma^{-1} H^{T} H & 0 & 0 \\
0 & -Q_{2} & 0 \\
0 & 0 & -\gamma I
\end{array}\right]+\left[\begin{array}{c}
A_{1 k}^{T} \\
A_{2 k}^{T} \\
G^{T}
\end{array}\right] P\left[\begin{array}{c}
A_{1 k}^{T} \\
A_{2 k}^{T} \\
G^{T}
\end{array}\right]^{T} } \\
& +\left[\begin{array}{ccc}
U_{1}+K_{1}^{T} U_{3} K_{1}+\gamma^{-1} H^{T} H & K_{1}^{T} U_{3} K_{2} & 0 \\
K_{2}^{T} U_{3} K_{1} & U_{2}+K_{2}^{T} U_{3} K_{2} & 0 \\
0 & 0 & 0
\end{array}\right] .
\end{aligned}
$$

Proof. Similar to Theorem 3.10, pre- and postmultiplying the left-hand side matrix in (3.38) by the matrix $\operatorname{diag}[P, P, I, I, I, I, I, I, I, I]$, respectively, using Lemmas 3.8 and 3.9 , it can be obtained that (3.38) is equivalent to $\bar{\Gamma}<0$. For $\left[\begin{array}{cc}U_{1}+K_{1}^{T} U_{3} K_{1} & K_{1}^{T} U_{3} K_{2} \\ K_{2}^{T} U_{3} K_{1} & U_{2}+K_{2}^{T} U_{3} K_{2}\end{array}\right]>0$, it implies $\sum_{t=0}^{M_{1}}$ $\sum_{k=1}^{M_{2}} J_{1} \leq 0$. In other words, if (3.38) holds, $\|z(t, k)\|_{2 \mathrm{D}-2 e} \leq \gamma\|\omega(t, k)\|_{2 \mathrm{D}-2 e}$ is guaranteed. This completes the proof of Theorem 3.11.

The closed-loop system (3.8) can be depicted by Figure 1, where the dotted arrowed lines denote the information flow of the last cycle from the storages, whereas the solid arrowed lines represent the flow of real-time feedback information. This block diagram can be explained from two different angles: one is as a $2 \mathrm{D}$ system; the other is as a batch process. From the 2D system point of view, the closed-loop system consists of a $2 \mathrm{D}$ model $\sum_{2 \mathrm{D}-e p-F}$ and a $2 \mathrm{D}$ state feedback controller $\sum_{C}$. Plant $\sum_{2 \mathrm{D}-e p-F}$ clearly is a $2 \mathrm{D}$ system that is composed of a repetitive process and an iterative loop. From the perspective of a batch process, the

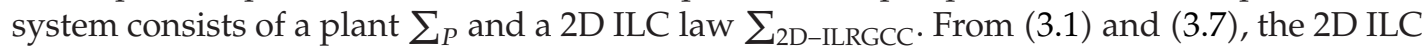
law $\sum_{\text {2D-ILRGCC }}$ can be decomposed as follows:

$$
\sum_{\text {2D-ILRGCC }}: u(t, k)=u_{l}(t, k-1)+u_{r}(t, k)
$$

where $u_{l}(t, k-1)=u(t, k-1)+K_{2} X(t+1, k-1)$ is an iterative learning control law for the performance improvement along the cycle direction, and $u_{r}(t, k)=K_{1} X(t, k)$ is a real-time state feedback control law for ensuring control performance over time.

\subsection{Procedure Design of the Controller}

From the definitions of $Q_{1}$ and $Q_{2}: Q_{1}=\lambda P, Q_{2}=(1-\lambda) P$, and Theorem 3.10, we have

$$
\begin{aligned}
J & \leq \sum_{k=1}^{N_{2}} X^{T}(0, k) P S_{1} P x X(0, k)+\sum_{t=0}^{N_{1}} X^{T}(t+1,0) P S_{2} P X(t+1,0) \\
& =\sum_{k=1}^{N_{2}} X^{T}(0, k) \lambda \Omega^{-1} X(0, k)+\sum_{t=0}^{N_{1}} X^{T}(t+1,0)(1-\lambda) \Omega^{-1} X(t+1,0) .
\end{aligned}
$$


Guaranted cost control for batch processes

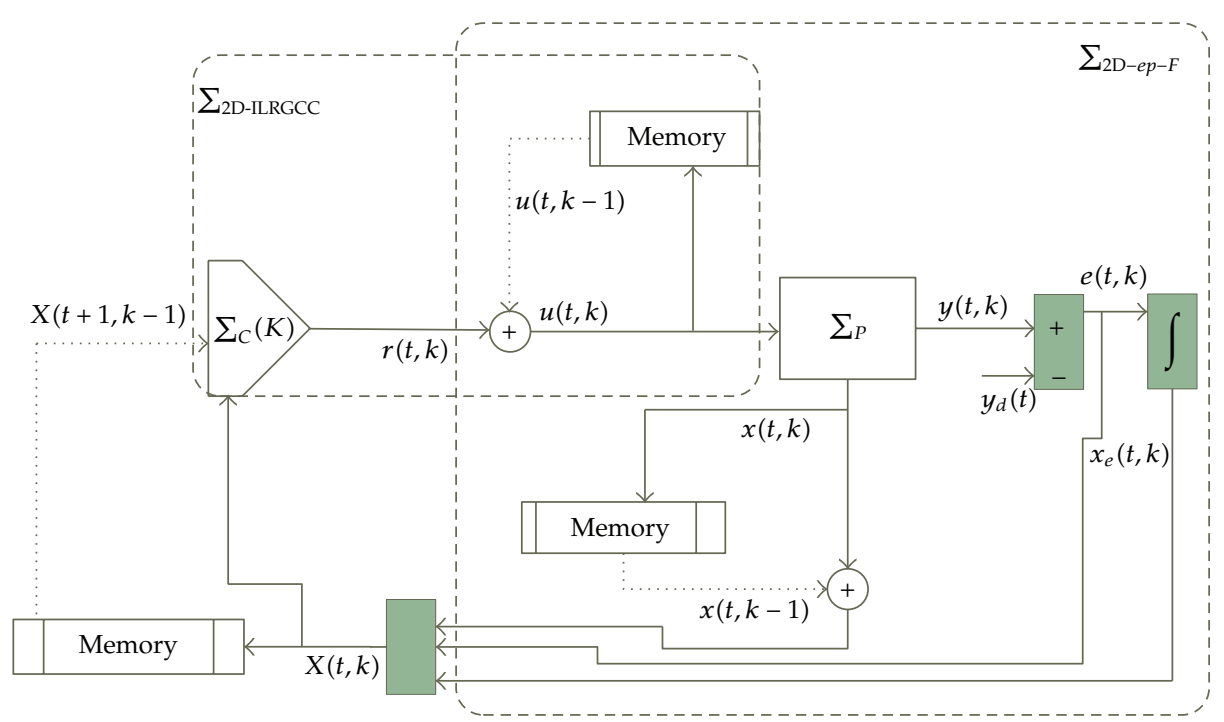

Figure 1: Schematic diagram of the structure of a closed-loop system.

Note that the upper bound of cost function in Theorem 3.10 depends on the initial conditions satisfying (3.5). For any $N_{1} \geq r_{1}$ and $N_{2} \geq r_{2}$, the boundary conditions satisfy (3.9a)-(3.9b). So the cost bound (3.46) leads to

$$
J \leq r_{2} \lambda \beta+r_{1}(1-\lambda) \beta,
$$

where

$$
\left[\begin{array}{cc}
-\beta I & \Theta^{T} \\
\Theta & -L
\end{array}\right]<0
$$

In order to obtain the controller $r(t, k)=Y_{1} L^{-1} X(t, k)+Y_{2} L^{-1} X(t+1, k-1)$ and achieves as far as possible the least guaranteed cost value $J^{*}$, we have to solve the following optimization problem:

$$
\begin{gathered}
\min \quad r_{2} \lambda \beta+r_{1}(1-\lambda) \beta \\
\text { s.t. } \quad 0<\lambda<1,(3.24),(3.48) .
\end{gathered}
$$

When $0<\lambda<1$ is given, the previous optimization problem is a convex optimization problem (3.49) which can be solved by the solver mincx in the LMI toolbox. Furthermore, we can find the optimal scalar $\lambda^{*}$ such that the guaranteed cost bound (3.46) is minimized. 
It can be seen from Theorem 3.11 that although the upper bound of cost function in Theorem 3.11 is similar to that in Theorem 3.10, the constraint condition is different from that in Theorem 3.10. The optimization problem is

$$
\begin{gathered}
\min \quad r_{2} \lambda \beta+r_{1}(1-\lambda) \beta \\
\text { s.t. } \quad 0<\lambda<1,(3.38),(3.48) .
\end{gathered}
$$

For the LMI that is described by (3.38), $\gamma>0$ should be regarded as an optimization variable, and the design objective is such that $\gamma$ is as small as possible. At the same time, we will find the optimal scalar $\lambda^{*}$ such that the guaranteed cost bound (3.50) is minimized. In this paper, in order to obtain the minimum guaranteed cost bound, there are no constraints to $\gamma$. The ideal $\lambda^{*}$ can be obtained by using the following method: given a larger $\lambda$, solve the inequalities (3.38). If there is a feasible solution, then given a smaller $\lambda$, go on; otherwise stop.

\section{Numerical Example}

Injection molding, which is a batch process, mainly consists of three phases: filling, packing, and cooling [9]. For the packing/holding phase, nozzle pressure is a key process variable that could be controlled to follow a preset profile to ensure product quality and consistency from cycle to cycle. Variations of working conditions may make injection molding particularly packing-holding viewed as a batch process with uncertain parameter perturbations. In each cycle, the transition of different phases, for example, from filling to packing/holding, leads to uncertain initial values of the nozzle pressure. This makes the conventional ILC not applicable. On the other hand, the control performance is typically poor when a slow hydraulic valve is used. Pure feedback control, such as PID (proportional integrative derivative) control and adaptive control, cannot improve control performance from cycle to cycle. It is necessary to design a robust and $\backslash$ or $H_{\infty} 2 \mathrm{D}$ controller that can ensure improving both the performances over time and the tracking performance from cycle to cycle. Based on the open-loop test and analysis, identifying the nozzle packing pressure response to the hydraulic control valve opening, the state-space model seen as the state variables [17] is adopted:

$$
\begin{gathered}
\sum_{P}: x(t+1, k)=\left(\left[\begin{array}{ccc}
1.607 & -0.6086 & 0.9282 \\
1 & 0 & 0 \\
0 & 0 & 0
\end{array}\right]+\Delta A\right) x(t, k)+\left[\begin{array}{c}
1.239 \\
0 \\
1
\end{array}\right] u(t, k), \\
y(t, k)=\left[\begin{array}{lll}
1 & 0 & 0
\end{array}\right] x(t, k),
\end{gathered}
$$

where parameter perturbations expressed as $E \Delta(t) F$ and $E \Delta(t) F_{b}$ are $E=\left[\begin{array}{ccc}0.1 & 0 & 0 \\ 0 & 0.1 & 0 \\ 0 & 0 & 0.1\end{array}\right], F=$ $\left[\begin{array}{ccc}0.104 & -0.204 & -0.264 \\ 0 & 0 & 0 \\ 0 & 0 & 0\end{array}\right], \Delta(t)=\left[\begin{array}{ccc}\delta_{1} & 0 & 0 \\ 0 & \delta_{2} & 0 \\ 0 & 0 & \delta_{3}\end{array}\right]$, and $\left|\delta_{i}\right| \leq 1, i=1,2,3$. Assume that there exists an unknown actuator failure $\alpha$; however, we know that $0.8=\underline{\alpha} \leq \alpha \leq \bar{\alpha}=1$. Using (2.6a)-(2.6c), $\beta=0.9$ and $\beta_{0}=0.1$ are obtained. Here the set-point takes the following form:

$$
\begin{array}{ll}
y_{d}(t)=15 & (\text { for } 0 \leq t \leq 100) \\
y_{d}(t)=30 & (\text { for } 100 \leq t \leq 200)
\end{array}
$$


The initial state satisfies condition (3.9a)-(3.9b) for $r_{1}=r_{2}=10$ and belongs to the set $\mathbb{S}\left(d_{M}, 0\right)$ where

$$
\Theta=\left[\begin{array}{ccc}
20 & 0 & 0 \\
0 & 20 & 0 \\
0 & 0 & 20
\end{array}\right]
$$

Choose the weighting matrices

$$
Q_{1}=Q_{2}=\left[\begin{array}{lll}
1 & 0 & 0 \\
0 & 1 & 0 \\
0 & 0 & 1
\end{array}\right], \quad U=1
$$

Case 1 (repetitive uncertainty). In this simulation case, we first consider repetitive parameter perturbations, that is, $\omega(t, k)=0$. For different $\lambda$, solve the optimization problem (3.49), fit the guaranteed cost $J^{*}$, and show them by Figure 2(a). When $\lambda=0.731$, the least upper bound of the corresponding closed-loop cost function is $J^{*}=1.6752 e+005$ for the resulting closed-loop system, and we obtain the optimal guaranteed cost controller as follows:

$$
\begin{aligned}
r^{*}(t, k+1)= & {\left[\begin{array}{llllll}
-1.4641 & 0.5996 & 0.9146 & -0.2080 & -0.2081
\end{array}\right] X(t, k) } \\
& +\left[\begin{array}{llllll}
0.0000 & -0.0000 & -0.0000 & 0.0000 & -0.8356
\end{array}\right] X(t+1, k-1)
\end{aligned}
$$

To show tracking results, we choose the extended optimal ILRGCC (4.5) to stabilize the system (4.1) and use the output tracking error in terms of root-sum-squared-error (REES) criterion. The results are shown in Figure 3(a); here $\left\{\delta_{i}:\left|\delta_{i}\right|<1\right\}_{i=1.2 .3}$ are assumed to vary randomly within $[0,1]$ along time direction. From Figure 3(a), we can see that the tracking performance is improved from cycle to cycle; although after fault occurs, the tracking performance experiences degradation, the tracking performance can achieve a perfect level again some cycles later and even restore to the original level.

Case 2 (nonrepetitive uncertainty). In this case, according to nonrepeatable perturbations $\left\{\delta_{i}:\left|\delta_{i}\right|<1\right\}_{i=1.2 .3}$ which are taken as random variables within $[0,1]$ along batch direction, we design a $\gamma$-optimal 2D $H_{\infty}$ ILRGCC for system (4.1). From the constraints, we can see that we must first continue to adjust a suitable value of $\lambda$ which will directly affect the optimal performance. On the other hand, the $H_{\infty}$-performance index, $\gamma$, will be obtained by solving the LMI (3.38), rather than by solving some constraints. Solving the optimization problem (3.50) for different $\lambda$, the corresponding guaranteed cost $J^{*}$ and $H_{\infty}$-performance index $\gamma$ via different $\lambda$ are shown in Figures 2(b) and 2(c), respectively. From the two figures we can see that although $J^{*}$ will be the least, $\gamma$ may be not the least one. This present paper is to design the $H_{\infty}$ guaranteed cost controller such that corresponding guaranteed cost $J^{*}$ is the least. So, when $\lambda=0.83$, the least guaranteed cost is $J^{*}=7.9856 e+005$. Correspondingly, $H_{\infty}$-performance index $\gamma$ equals 899.0908, and the optimal guaranteed cost controller is

$$
\begin{aligned}
r^{*}(t, k+1)= & {\left[\begin{array}{lllll}
-1.3490 & 0.5286 & 0.8062 & -0.1910 & -0.1910
\end{array}\right] X(t, k) } \\
& +\left[\begin{array}{lllll}
-0.0000 & -0.0000 & -0.0000 & -0.0000 & -0.8100
\end{array}\right] X(t+1, k-1) .
\end{aligned}
$$




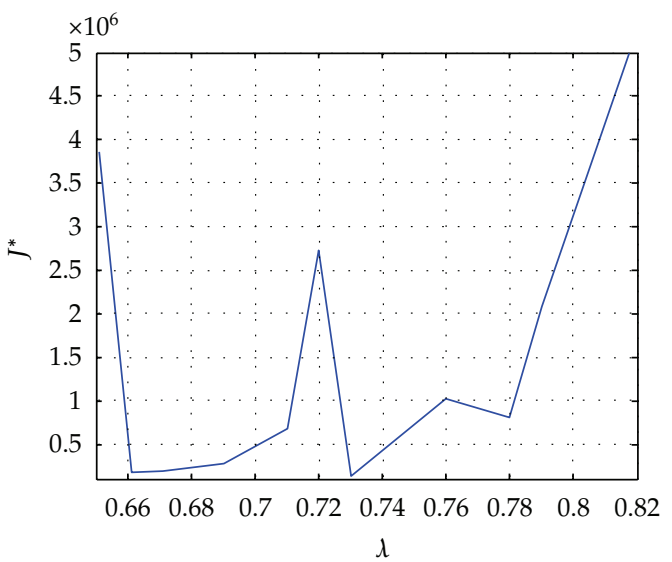

(a)

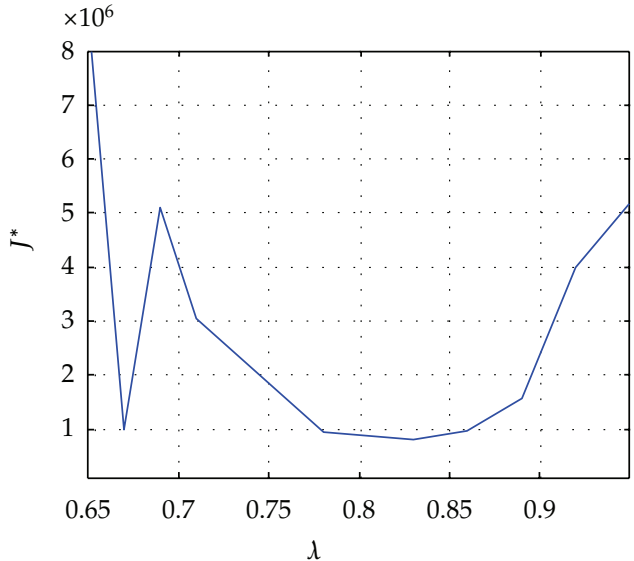

(b)

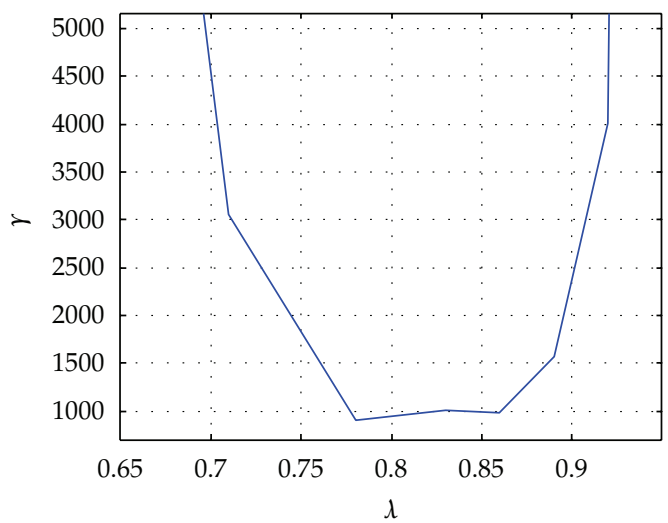

(c)

Figure 2: (a) The guaranteed cost $J^{*}$ via different $\lambda$. (b) The guaranteed cost $J^{*}$ via different $\lambda$ for every corresponding solvable $\gamma$. (c) Different $\lambda$ for every corresponding solvable $\gamma$.

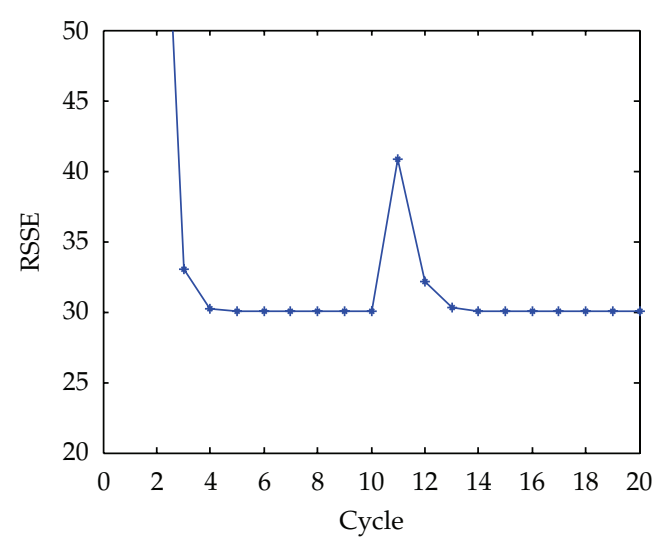

(a)

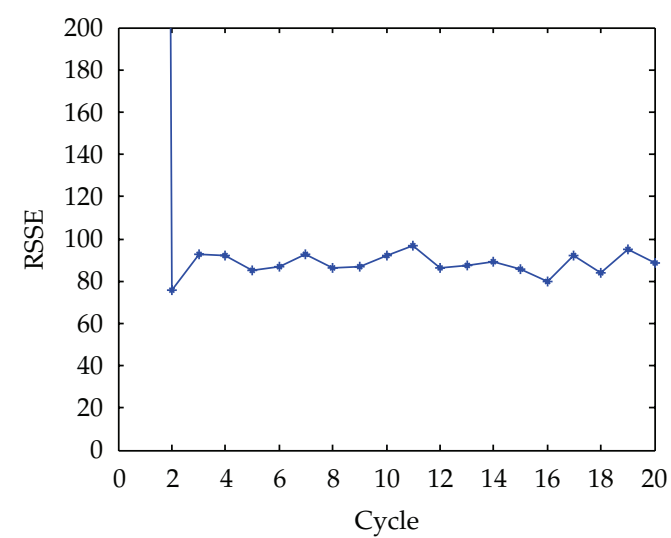

(b)

Figure 3: (a) Tracking performances in Case 1. (b) Tracking performances in Case 2. 
From these results it can be distinctly seen that the system performance is indeed decreased when the system is affected by the interference. Figure 3(b) also shows the effect. It is seen that the proposed method holds the good robustness and convergence of the designed control system against some degree of nonrepetitive uncertainty by using controller (4.6) even though the system performance is decreased.

\section{Conclusion}

By an LMI framework, the optimal fault-tolerant guaranteed cost control problem via a robust and $\backslash$ or $H_{\infty}$ 2D-ILRGCC has been proposed for a batch process. The process has been transformed to an equivalent 2D-FM model, based on which relevant concepts on the optimal guaranteed cost control design with extended information have been presented. Through solving the corresponding LMI constraints, the optimal robust and $\backslash$ or $H_{\infty} 2 \mathrm{D}$ guaranteed cost controller has been explicitly formulated, together with preserving robust control performance levels. The proposed robust and $\backslash$ or $H_{\infty}$ 2D-LRGCC can have control performance improvement not only along the time direction but also along the cycle direction, under the normal case as well as the actuator fault case. An injection pressure control has been developed to demonstrate the effectiveness and merits of the proposed ILC method.

\section{Acknowledgments}

This work is supported in part by NSFC/RGC joint Research Scheme (under Project no. N-HKUST639/09), NSFC (through Project nos. 60931160440, 61104058), and Guangzhou Scientific and Technological Project (through Project no. 11F11140010).

\section{References}

[1] Anon, "Automatic control of batch-process temperature," Food Industries, vol. 8, no. 5, pp. 237-244, 1936.

[2] E. Korovessi and A. A. Linninger, Batch Processes, CRC/Taylor and Francis, Boca Raton, Fla, USA, 2006.

[3] D. Ye and G. H. Yang, "Delay-dependent reliable Hœ control for linear time-varying delay systems via adaptive approach," in American Control Conference (ACC '07), pp. 3991-3996, July 2007.

[4] D. Yue and J. Lam, "Reliable memory feedback design for a class of non-linear time-delay systems," International Journal of Robust and Nonlinear Control, vol. 14, no. 1, pp. 39-60, 2004.

[5] D. Yue, J. Lam, and D. W. C. Ho, "Reliable Ho control of uncertain descriptor systems with multiple time delays," IEE Proceedings: Control Theory and Applications, vol. 150, no. 6, pp. 557-564, 2003.

[6] Y. Wang, J. Shi, D. Zhou, and F. Gao, "Iterative learning fault-tolerant control for batch processes," Industrial and Engineering Chemistry Research, vol. 45, no. 26, pp. 9050-9060, 2006.

[7] Y. Wang, D. Zhou, and F. Gao, "Iterative learning reliable control of batch processes with sensor faults," Chemical Engineering Science, vol. 63, no. 4, pp. 1039-1051, 2008.

[8] Y. Wang, Y. Yang, D. Zhou, and F. Gao, "Active fault-tolerant control of nonlinear batch processes with sensor faults," Industrial and Engineering Chemistry Research, vol. 46, no. 26, pp. 9158-9169, 2007.

[9] F. Gao, Y. Yang, and C. Shao, "Robust iterative learning control with applications to injection molding process," Chemical Engineering Science, vol. 56, no. 24, pp. 7025-7034, 2001.

[10] D. Gorinevsky, "Loop shaping for iterative control of batch processes," IEEE Control Systems Magazine, vol. 22, no. 6, pp. 55-65, 2002.

[11] Z. Xiong and J. Zhang, "Product quality trajectory tracking in batch processes using iterative learning control based time-varying perturbation models," Industrial and Engineering Chemistry Research, vol. 42, no. 26, pp. 6802-6814, 2003. 
[12] J. Shi, F. Gao, and T. J. Wu, "From two-dimensional linear quadratic optimal control to iterative learning control. Paper 1. Two-dimensional linear quadratic optimal controls and system analysis," Industrial and Engineering Chemistry Research, vol. 45, no. 13, pp. 4603-4616, 2006.

[13] J. Shi, F. Gao, and T. J. Wu, "From two-dimensional linear quadratic optimal control to iterative learning control. Paper 2. Iterative learning controls for batch processes," Industrial and Engineering Chemistry Research, vol. 45, no. 13, pp. 4617-4628, 2006.

[14] J. Shi, F. Gao, and T. J. Wu, "Single-cycle and multi-cycle generalized 2D model predictive iterative learning control (2D-GPILC) schemes for batch processes," Journal of Process Control, vol. 17, no. 9, pp. 715-727, 2007.

[15] J. Shi, F. Gao, and T. J. Wu, "Robust design of integrated feedback and iterative learning control of a batch process based on a 2D Roesser system," Journal of Process Control, vol. 15, no. 8, pp. 907-924, 2005.

[16] J. Shi, F. Gao, and T. J. Wu, “Robust iterative learning control design for batch processes with uncertain perturbations and initialization," AIChE Journal, vol. 52, no. 6, pp. 2171-2187, 2006.

[17] J. Shi, F. Gao, and T. J. Wu, "Integrated design and structure analysis of robust Iterative learning control system based on a two-dimensional model," Industrial and Engineering Chemistry Research, vol. 44, no. 21, pp. 8095-8105, 2005.

[18] S. S. L. Chang and T. K. C. Peng, "Adaptive guaranteed cost control of systems with uncertain parameters," IEEE Transactions on Automatic Control, vol. 17, no. 4, pp. 474-483, 1972.

[19] A. Dhawan and H. Kar, "Optimal guaranteed cost control of 2-D discrete uncertain systems: an LMI approach," Signal Processing, vol. 87, no. 12, pp. 3075-3085, 2007.

[20] A. Dhawan and H. Kar, "An lmi approach to robust optimal guaranteed cost control of 2-d discrete systems described by the roesser model," Signal Processing, vol. 90, no. 9, pp. 2648-2654, 2010.

[21] A. Dhawan and H. Kar, "An improved LMI-based criterion for the design of optimal guaranteed cost controller for 2-D discrete uncertain systems," Signal Processing, vol. 91, no. 4, pp. 1032-1035, 2011.

[22] I. R. Petersen, "A stabilization algorithm for a class of uncertain linear systems," Systems $\mathcal{E}$ Control Letters, vol. 8, no. 4, pp. 351-357, 1987.

[23] S. Boyd, L. E. Ghaoui, E. Feron et al., Linear Matrix Inequality in System and Control Theory, SIAM Studies in Applied Mathematics, SIAM, Philadelphia, Pa, USA, 1994. 


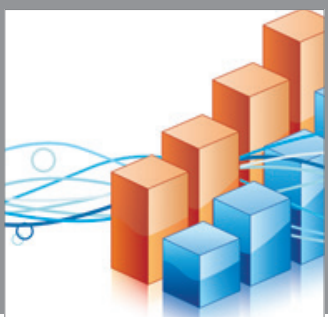

Advances in

Operations Research

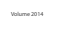

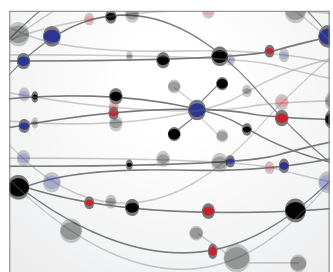

\section{The Scientific} World Journal
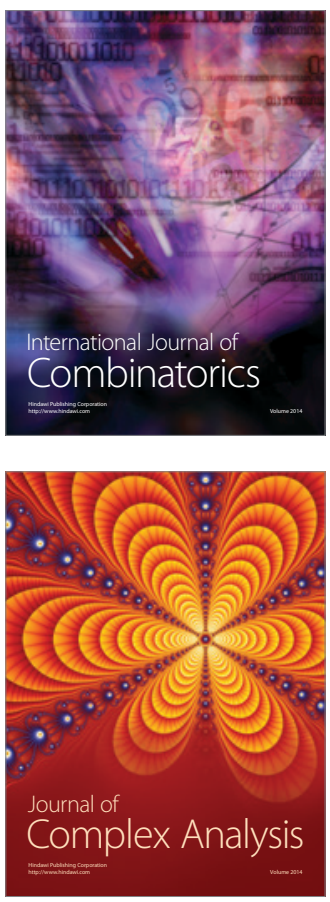

International Journal of

Mathematics and

Mathematical

Sciences
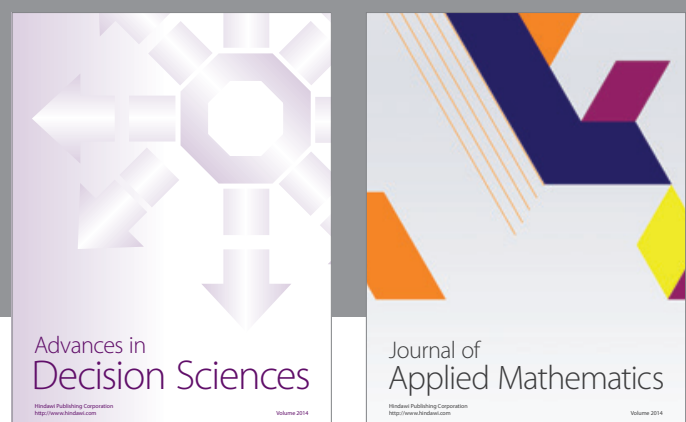

Journal of

Applied Mathematics
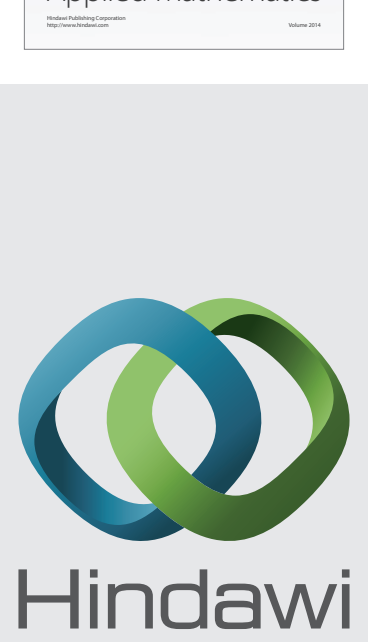

Submit your manuscripts at http://www.hindawi.com
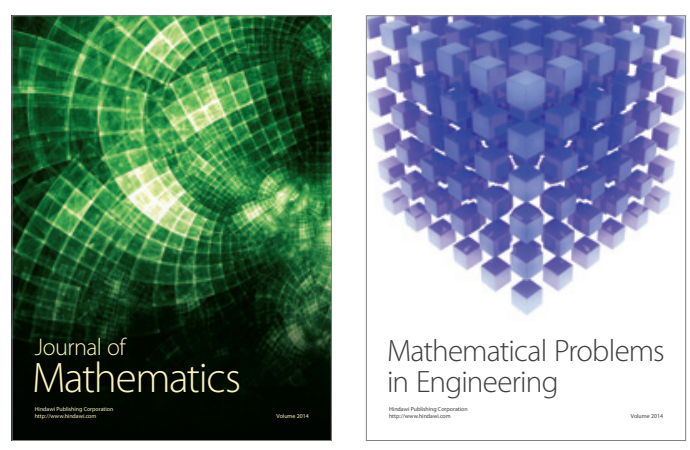

Mathematical Problems in Engineering
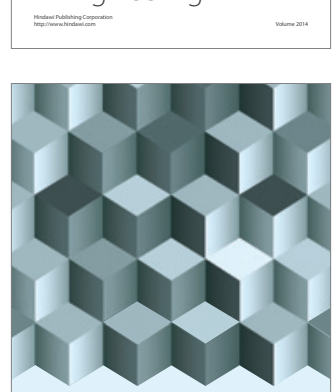

Journal of

Function Spaces
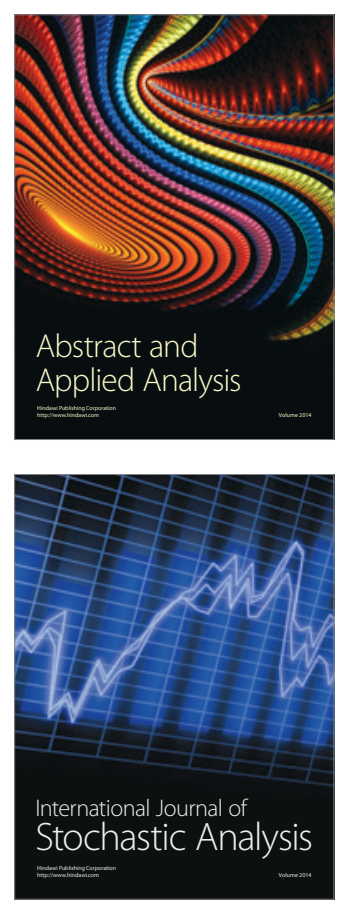

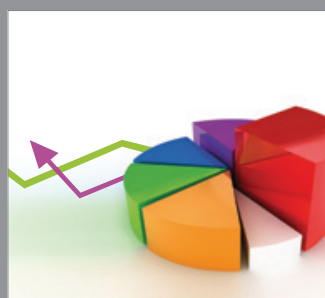

ournal of

Probability and Statistics

Promensencen
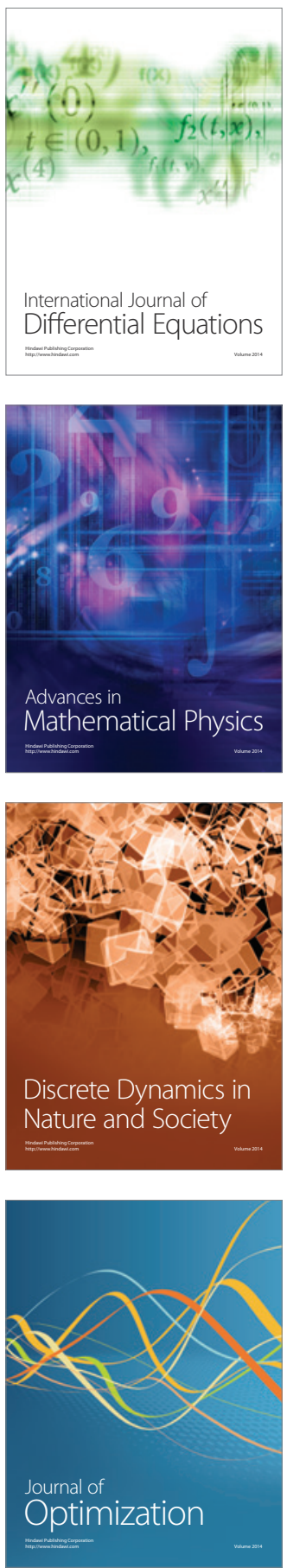